\title{
SULLA DETERMINAZIONE DI TUTTE LE SUPERFICIE APPLICABILI SU DI UNA SUPERFICIE DATA.
}

Nota di Ubaldo Barbieri, in Roma.

Adunanza del 23 giugno 1901.

E noto come i primi lavori su questo importante e difficile soggetto cominciarono ad aversi dopo il I860, nel quale anno l'Accademia delle Scienze di Parigi nella seduta del 9 luglio, bandiva un gran premio di matematiche "sulla questione concernente le superficie applicabili su di una superficie data".

Tre memorie, quella del Bour ${ }^{*}$ ), quella del Codazzi ${ }^{* *}$ ) e quella del Bonnet ${ }^{* *}$ ) furono ritenute degne di maggior considerazione sulle altre; la prima vinse il premio, la seconda ottenne una menzione onorevole.

Esse furono, per cosi dire, tre memorie classiche e, forse, contribuirono agli altri susseguenti lavori del Darboux, del Weingarten, dell'Halphen, del Lie, del Goursat e del Liouville $\dagger$ ), sino al i 896 .

Non diru di tali lavori, non essendo questo il còmpito del presente

*) E. Bour, Theorie de la déformation des surfaces [Journal de l'École Polytechnique, $\mathrm{XXXIX}^{\mathrm{e}}$ Cahier (1862)].

**) D. Codazzi, Mémoire relatif à l'application des surfaces les unes sur les autres, envoyé au Concours ouvert sur cette question en 1860 par l'Académie des Sciences [t. XXVII des Mémoires présentées par divers savants à l'Académie des Sciences (1882)].

***) O. Bonnet, Mémoire sur les surfaces applicables [Journal de l'École Polytechnique, XIII Cahier (1867)].

f) Per tutti questi lavori, vedi: G. Darboux, Théorie générale des surfaces, tomes III et IV. 
studio: tuttavia, rammenterò come sino al 1896 si fosse solo giunti a far dipendere la soluzione del problema da un'equazione alle derivate parziali del secondo ordine, del tipo di quelle d'AMpère, ma non integrabile che per le superficie sviluppabili: $i$ pochi casi risolti interamente lo erano stati per via indiretta. Inoltre, tale equazione presentava il grave inconveniente che non tutte le sue soluzioni reali erano soluzioni del problema.

Ma pochi anni or sono, nel I896 appunto, il sig. Giulio WernGARTEN pubblicava una memoria di eccezionale importanza, che riportava il gran premio nel concorso indetto dall'Accademia delle Scienze di Parigi nel 1894 .

In tale memoria, com'è noto, egli giunge ad un'equazione eziandio alle derivate parziali del $2^{\circ}$ ordine e del tipo d'AMPÉre: peraltro, riesce ad integrarla nel caso generale del paraboloide di rivoluzione, ed in alcuni altri casi particolari, che lo portano ai risultati conosciuti antecedentemente per altre vie. Per di più, tutte le soluzioni reali di essa equazione sono soluzioni del problema.

Ma l'illustre geometra tedesco non ha reso noti i criterii razionali che lo hanno guidato nella sua ricerca: "Il se propose, donc, de cher"cher par une voie différente de toutes celles qui sont connues, une ré"duction nouvelle du problème à l'intégration d'une équation aux déri"vées partielles du second ordre, et il obtient le but qu'il s'est proposé, " a l'aide d'une méthode qui nous a paru extrêmement ingénieuse, bien "que les principes sur lesquels elle repose ne soient pas complètement " mis en évidence par l'exposition"*).

Scopo del presente lavoro è, appunto, quello di ottenere gli stessi risultati con procedimento più facile, più razionale e che ponga in rilievo l'utilita di certe posizioni e di certi artifizii.

Alla fine di questo studio mostro, eziandio, come direttamente derivi dal procedimento analitico da me seguito, l'elegante interpretazione geometrica che il sig. Darboux ha: dato del metodo del Weingarten nel $\mathrm{IV}^{\circ}$ volume della sua "Théorie générale des surfaces».

*) Vedi: Comptes rendus de l'Académie des Sciences de Paris, tome CXIX (1894), pag. Ioso. 
Sia dato l'elemento lineare di una superficie $S$ :

$$
d s=\sqrt{E d z^{2}+2 F d z d \sigma+G d \sigma^{2}}
$$

e si vogliano determinare tutte le superficie che ammettono questo dato elemento lineare.

Siano $\xi, n, \zeta$ le coordinate di un punto qualunque di una superficie $S_{1}$ soddisfacente a tale condizione: sarà allora verificata l'uguaglianza

$$
d \xi^{2}+d n^{2}+d \zeta^{2}=E d z^{2}+2 F d z d \sigma+G d \sigma^{2} .
$$

Poniamo

senza dir altro per ora.

$$
\left\{\begin{array}{l}
E=a^{2}+b^{2} \\
F=a \alpha+b \beta \\
G=\alpha^{2}+\beta^{2}
\end{array}\right.
$$

$\mathrm{La}$ (I) diverrà di conseguenza

(3) $d \xi^{2}+d n^{2}+d \zeta^{2}=\left(a^{2}+b^{2}\right) d z^{2}+2(a \alpha+b \beta) d z d \sigma+\left(\alpha^{2}+\beta^{2}\right) d \sigma^{2}$.

Affinchè soddisfino, basterd che $d \xi, d n, d \zeta$ abbiano la forma:

(4)

$$
\left\{\begin{array}{l}
d \xi=\left(a X+b X^{\prime}\right) d z+\left(\alpha X+\beta X^{\prime}\right) d \sigma, \\
d n=\left(a Y+b Y^{\prime}\right) d z+\left(\alpha Y+\beta Y^{\prime}\right) d \sigma, \\
d \zeta=\left(a Z+b Z^{\prime}\right) d z+\left(\alpha Z+\beta Z^{\prime}\right) d \sigma,
\end{array}\right.
$$

dove $X, Y, Z ; X^{\prime}, Y^{\prime}, Z^{\prime}$ rappresentino $\mathrm{i}$ coseni di due direzioni ortogonali tra loro, ossia soddisfino alle relazioni:

(5)

$$
\left\{\begin{array}{l}
X^{2}+Y^{2}+Z^{2}=\mathrm{I} \\
X^{\prime 2}+Y^{\prime 2}+Z^{\prime 2}=\mathrm{I} \\
X X^{\prime}+Y Y^{\prime}+Z Z^{\prime}=0 .
\end{array}\right.
$$

Esprimiamo le condizioni d'integrabilità delle (4); esse sono:

(6)

$$
\left\{\begin{array}{l}
\frac{\partial\left(a X+b X^{\prime}\right)}{\partial \sigma}=\frac{\partial\left(\alpha X+\beta X^{\prime}\right)}{\partial z}, \\
\frac{\partial\left(a Y+b Y^{\prime}\right)}{\partial \sigma}=\frac{\partial\left(\alpha Y+\beta Y^{\prime}\right)}{\partial z}, \\
\frac{\partial\left(a Z+b Z^{\prime}\right)}{\partial \sigma}=\frac{\partial\left(\alpha Z+\beta Z^{\prime}\right)}{\partial z} .
\end{array}\right.
$$

Inoltre, dalle (4) ancora, osservando che $d \xi=\frac{\partial \xi}{\partial z} d z+\frac{\partial \xi}{\partial \sigma} d \sigma$; 
$d n=$ etc. ricaviamo immediatamente $\mathrm{i}$ valori:

(7)

$$
\left\{\begin{array} { l } 
{ X = \frac { \beta \frac { \partial \xi } { \partial z } - b \frac { \partial \xi } { \partial \sigma } , } { \sqrt { \Delta } } , } \\
{ Y = \frac { \beta \frac { \partial \eta } { \partial z } - b \frac { \partial n } { \partial \sigma } } { \sqrt { \Delta } } , } \\
{ Z = \frac { \beta \frac { \partial \zeta } { \partial z } - b \frac { \partial \zeta } { \partial \sigma } , } { \sqrt { \Delta } } , }
\end{array} \left\{\begin{array}{l}
X^{\prime}=\frac{a \frac{\partial \xi}{\partial \sigma}-\alpha \frac{\partial \xi}{\partial z}}{\sqrt{\Delta}}, \\
Y^{\prime}=\frac{a \frac{\partial \eta}{\partial \sigma}-\alpha \frac{\partial \eta}{\partial z}}{\sqrt{\Delta}}, \\
Z^{\prime}=\frac{a \frac{\partial \zeta}{\partial \sigma}-\alpha \frac{\partial \zeta}{\partial z}}{\sqrt{\Delta}}
\end{array}\right.\right.
$$

(dove $\sqrt{\Delta}=a \beta-b x$ ), che coincidono con le espressioni (8) della memoria del Weingarten.

Tali valori di $X, Y, Z ; X^{\prime}, Y^{\prime}, Z^{\prime}$, giova osservarlo, presuppongono che $d \xi, d n, d \zeta$ siano differenziali esatti, $\mathrm{o}$, anche, che la superficie $S_{1}, \mathrm{i}$ cui punti hanno le coordinate correnti $\xi, n, \zeta$, sia applicabile sulla superficie data $S$.

Se a tali valori (7) aggiungiamo i noti valori dei coseni $X^{\prime \prime}, Y^{\prime \prime}, Z^{\prime \prime}$, della normale alla superficie nel punto $(\xi, n, \zeta)$ :

$\left(7^{\prime}\right)$

$$
\left\{\begin{aligned}
X^{\prime \prime} & =\frac{\frac{\partial n}{\partial z} \frac{\partial \zeta}{\partial \sigma}-\frac{\partial \zeta}{\partial z} \frac{\partial n}{\partial \sigma}}{\sqrt{\Delta}}, \\
Y^{\prime \prime} & =\frac{\frac{\partial \zeta}{\partial z} \frac{\partial \xi}{\partial \sigma}-\frac{\partial \xi}{\partial z} \frac{\partial \zeta}{\partial \sigma}}{\sqrt{\Delta}}, \\
Z^{\prime \prime} & =\frac{\frac{\partial \xi}{\partial z} \frac{\partial n}{\partial \sigma}-\frac{\partial n}{\partial z} \frac{\partial \xi}{\partial \sigma}}{\sqrt{\Delta}},
\end{aligned}\right.
$$

sarà facile verificare l'esistenza delle due relazioni:

$$
\left\{\begin{array}{l}
X X^{\prime \prime}+Y Y^{\prime \prime}+Z Z^{\prime \prime}=0 \\
X^{\prime} X^{\prime \prime}+Y^{\prime} Y^{\prime \prime}+Z^{\prime} Z^{\prime \prime}=0
\end{array}\right.
$$

Consegue che le tre direzioni $(X Y Z),\left(X^{\prime} Y^{\prime} Z^{\prime}\right),\left(X^{\prime \prime} Y^{\prime \prime} Z^{\prime \prime}\right)$ formano un triedro trirettangolo, essendo $\left(X^{\prime \prime} Y^{\prime \prime} Z^{\prime \prime}\right)$ i coseni della normale ed $\left(X^{\prime} Y^{\prime} Z^{\prime}\right),(X Y Z)$ quelli di due tangenti nel punto $(\xi, n, \zeta)$ di una superficie $S_{1}$ applicabile sulla superficie data $S$, l'elemento lineare della 
quale sia espresso da

$$
\sqrt{\left(a^{2}+b^{2}\right) d z^{2}+2(a \alpha+b \beta) d z d \sigma+\left(\alpha^{2}+\beta^{2}\right) d \sigma^{2}} \text {. }
$$

Quanto alle $a, b, \alpha, \beta$ esse sono funzioni delle variabili $z$ e $\sigma$; ma le equazioni :

$$
\left\{\begin{array}{l}
E=a^{2}+b^{2}, \\
F=a \alpha+b \beta, \\
G=\alpha^{2}+\beta^{2},
\end{array}\right.
$$

alle quali noi le abbiamo obligate a soddisfare, non bastano, evidentemente, per determinarle.

$\mathrm{La}$ determinazione completa delle quatiro funzioni $a, \alpha, b, \beta$ la stabiliremo nel corso delle successive operazioni, obligando le anzidette funzioni a soddisfare a quella relazione che più agevolerà i calcoli.

Per quanto precede, possiamo dire che:

"La determinazione di tutte le superficie aventi l'elemento lineare

$$
\sqrt{\left(a^{2}+b^{2}\right) d z^{2}+2(a z+b \beta) d z d \sigma+\left(\alpha^{2}+\beta^{2}\right) d \sigma^{2}}
$$

sarà ottenuta con la determinazione delle sei funzioni $X, Y, Z ; X^{\prime}, Y^{\prime}, Z^{\prime}$ di z e $\sigma$, tali da soddisfare alle equazioni (5) e (6)».

Queste relazioni noi le possiamo scrivere cosi:

(9)

$$
\begin{aligned}
& X\left(\frac{\partial a}{\partial \sigma}-\frac{\partial \alpha}{\partial z}\right)+\frac{\partial X}{\partial \sigma} a-\frac{\partial X}{\partial z} \alpha+X^{\prime}\left(\frac{\partial b}{\partial \sigma}-\frac{\partial \beta}{\partial z}\right)+\frac{\partial X^{\prime}}{\partial \sigma} b-\frac{\partial X^{\prime}}{\partial z} \beta=0, \\
& Y\left(\frac{\partial a}{\partial \sigma}-\frac{\partial x}{\partial z}\right)+\frac{\partial Y}{\partial \sigma} a-\frac{\partial Y}{\partial z} \alpha+Y^{\prime}\left(\frac{\partial b}{\partial \sigma}-\frac{\partial \beta}{\partial z}\right)+\frac{\partial Y^{\prime}}{\partial \sigma} b-\frac{\partial Y^{\prime}}{\partial z} \beta=0, \\
& Z\left(\frac{\partial a}{\partial \sigma}-\frac{\partial \alpha}{\partial z}\right)+\frac{\partial Z}{\partial \sigma} a-\frac{\partial Z}{\partial z} \alpha+Z^{\prime}\left(\frac{\partial b}{\partial \sigma}-\frac{\partial \beta}{\partial z}\right)+\frac{\partial Z^{\prime}}{\partial \sigma} b-\frac{\partial Z^{\prime}}{\partial z} \beta=0, \\
& \text { (o) }\left\{\begin{array}{l}
X^{2}+Y^{2}+Z^{2}=\mathrm{I}, \\
X^{\prime 2}+Y^{\prime 2}+Z^{\prime 2}=\mathrm{I}, \\
X X^{\prime}+Y Y^{\prime}+Z Z^{\prime}=0,
\end{array}\right. \\
& \text { (I) }\left\{\begin{array} { l } 
{ \sum X \frac { \partial X } { \partial \sigma } = 0 } \\
{ \sum X \frac { \partial X } { \partial z } = 0 } \\
{ \sum ( X \frac { \partial X ^ { \prime } } { \partial \sigma } + X ^ { \prime } \frac { \partial X } { \partial \sigma } ) = 0 }
\end{array} \text { (II) } \left\{\begin{array}{l}
\sum X^{\prime} \frac{\partial X^{\prime}}{\partial \sigma}=0 \\
\sum X^{\prime} \frac{\partial X^{\prime}}{\partial z}=0 \\
\sum\left(X \frac{\partial X^{\prime}}{\partial z}+X^{\prime} \frac{\partial X}{\partial z}\right)=0,
\end{array}\right.\right.
\end{aligned}
$$

dove $\sum$ è simbolo di sommatoria estesa ad $X, Y, Z ; X^{\prime}, Y^{\prime}, Z^{\prime}$. 
Moltiplichiamo la prima equazione del sistema (9) per $X$, la seconda per $Y$, la terza per $Z$ e sommiamo; poi la prima per $X^{\prime}$, la seconda per $Y^{\prime}$, la terza per $Z^{\prime}$ e sommiamo ancora; tenendo allora conto delle (o) avremo subito:

$$
\begin{aligned}
& \frac{\partial a}{\partial \sigma}-\frac{\partial \alpha}{\partial z}+b \sum X \frac{\partial X^{\prime}}{\partial \sigma}-\beta \sum X \frac{\partial X^{\prime}}{\partial z}=0, \\
& \frac{\partial b}{\partial \sigma}-\frac{\partial \beta}{\partial z}+a \sum X^{\prime} \frac{\partial X}{\partial \sigma}-\alpha \sum X^{\prime} \frac{\partial X}{\partial z}=0,
\end{aligned}
$$

donde si trae:

$$
\begin{aligned}
& a\left(\frac{\partial a}{\partial \sigma}-\frac{\partial x}{\partial z}\right)+b\left(\frac{\partial b}{\partial \sigma}-\frac{\partial \beta}{\partial z}\right)-a \beta \sum X \frac{\partial X^{\prime}}{\partial z}-\alpha b \sum X^{\prime} \frac{\partial X}{\partial z}=0 \\
& \alpha\left(\frac{\partial a}{\partial \sigma}-\frac{\partial \alpha}{\partial z}\right)+\beta\left(\frac{\partial b}{\partial \sigma}-\frac{\partial \beta}{\partial z}\right)+\alpha b \sum X \frac{\partial X^{\prime}}{\partial \sigma}+a \beta \sum X^{\prime} \frac{\partial X}{\partial \sigma}=0 ;
\end{aligned}
$$

e da queste ultime, tenendo presente la terza delle (I) e delle (II):

$$
\left\{\begin{array}{l}
\sum X \frac{\partial X^{\prime}}{\partial z}=\frac{1}{a \beta-b \alpha}\left[a\left(\frac{\partial a}{\partial \sigma}-\frac{\partial \alpha}{\partial z}\right)+b\left(\frac{\partial b}{\partial \sigma}-\frac{\partial \beta}{\partial z}\right)\right]=-\sum X^{\prime} \frac{\partial X}{\partial z} \\
\sum X \frac{\partial X^{\prime}}{\partial \sigma}=\frac{1}{a \beta-b \alpha}\left[\alpha\left(\frac{\partial a}{\partial \sigma}-\frac{\partial \alpha}{\partial z}\right)+\beta\left(\frac{\partial b}{\partial \sigma}-\frac{\partial \beta}{\partial z}\right)\right]=-\sum X^{\prime} \frac{\partial X}{\partial \sigma}
\end{array}\right.
$$

Si vede subito l'utilità di vincolare $a, b, \alpha, \beta$ all'ulteriore relazione

$$
\alpha\left(\frac{\partial a}{\partial \sigma}-\frac{\partial \alpha}{\partial z}\right)+\beta\left(\frac{\partial b}{\partial \sigma}-\frac{\partial \beta}{\partial z}\right)=0 .
$$

In tal guisa $a, b, \alpha, \beta$ risultano perfettamente deterninate.

Dalla (I I) si trae allora

(I 2)

$$
\left\{\begin{array}{l}
\frac{\partial a}{\partial \sigma}-\frac{\partial \alpha}{\partial z}=\lambda \beta, \\
\frac{\partial b}{\partial \sigma}-\frac{\partial \beta}{\partial z}=-\lambda \alpha,
\end{array}\right.
$$

dove $\lambda$ è, dunque, funzione nota. Tali ulime equazioni coincidono, per quanto si vedrà in seguito, con le (6) del Weingarten.

Dalle tre prime equazioni del sistema (9) avremo, sostituendo,

$$
\left(g^{\prime}\right)\left\{\begin{array}{l}
\lambda \beta X+a \frac{\partial X}{\partial \sigma}-\alpha \frac{\partial X}{\partial z}-\lambda \alpha X^{\prime}+b \frac{\partial X^{\prime}}{\partial \sigma}-\beta \frac{\partial X^{\prime}}{\partial z}=0, \\
\lambda \beta Y+a \frac{\partial Y}{\partial \sigma}-\alpha \frac{\partial Y}{\partial z}-\lambda \alpha Y^{\prime}+b \frac{\partial Y^{\prime}}{\partial \sigma}-\beta \frac{\partial Y^{\prime}}{\partial z}=0, \\
\lambda \beta Z+a \frac{\partial Z}{\partial \sigma}-\alpha \frac{\partial Z}{\partial z}-\lambda \alpha Z^{\prime}+b \frac{\partial Z^{\prime}}{\partial \sigma}-\beta \frac{\partial Z^{\prime}}{\partial z}=0 .
\end{array}\right.
$$


A questo sistema conviene dare un'altra forma.

Cominciamo dall'esprimerci $X^{\prime}, Y^{\prime}, Z^{\prime}$ in funzione di $X, Y, Z$ e delle derivate di queste.

In virtù delle ( $\mathrm{I}$ o) e delle ( $\mathrm{I}$ I) potremo a questo scopo considerare il sistema

$$
\left\{\begin{array}{l}
\sum X^{\prime} \frac{\partial X}{\partial z}=-\lambda \\
\sum X^{\prime} \frac{\partial X}{\partial \sigma}=0 \\
\sum X X^{\prime}=0
\end{array}\right.
$$

donde si traggono i valori:

essendo

$$
\left\{\begin{array}{l}
X^{\prime}=-\frac{\lambda}{D}\left(\frac{\partial Y}{\partial \sigma} Z-Y \frac{\partial Z}{\partial \sigma}\right) \\
Y^{\prime}=-\frac{\lambda}{D}\left(\frac{\partial Z}{\partial \sigma} X-Z \frac{\partial X}{\partial \sigma}\right) \\
Z^{\prime}=-\frac{\lambda}{D}\left(\frac{\partial X}{\partial \sigma} Y-X \frac{\partial Y}{\partial \sigma}\right)
\end{array}\right.
$$

$$
D=\left|\begin{array}{ccc}
\frac{\partial X}{\partial z} & \frac{\partial Y}{\partial z} & \frac{\partial Z}{\partial z} \\
\frac{\partial X}{\partial \sigma} & \frac{\partial Y}{\partial \sigma} & \frac{\partial Z}{\partial \sigma} \\
X & Y & Z
\end{array}\right| .
$$

Analogamente, considerando il sistema:

(IS)

avremo

$$
\left\{\begin{array}{l}
\sum X \frac{\partial X^{\prime}}{\partial z}=\lambda \\
\sum X \frac{\partial X^{\prime}}{\partial \sigma}=0 \\
\sum X X^{\prime}=0
\end{array}\right.
$$

$$
\left\{\begin{array}{l}
X=\frac{\lambda}{D^{\prime}}\left(Z^{\prime} \frac{\partial Y^{\prime}}{\partial \sigma}-Y^{\prime} \frac{\partial Z^{\prime}}{\partial \sigma}\right) \\
Y=\frac{\lambda}{D^{\prime}}\left(X^{\prime} \frac{\partial Z^{\prime}}{\partial \sigma}-Z^{\prime} \frac{\partial X^{\prime}}{\partial \sigma}\right) \\
Z=\frac{\lambda}{D^{\prime}}\left(Y^{\prime} \frac{\partial X^{\prime}}{\partial \sigma}-X^{\prime} \frac{\partial Y^{\prime}}{\partial \sigma}\right)
\end{array}\right.
$$

dove il $D^{\prime}$ è espressione analoga al $D$. 
Cerchiamo ora di ricavarci dei valori per le derivate parziali di $X, Y, Z ; X^{\prime}, Y^{\prime}, Z^{\prime}$.

Per questo, unitamente alle due direzioni $(X, Y, Z),\left(X^{\prime}, Y^{\prime}, Z^{\prime}\right)$ ortogonali tra loro, torna utile considerarne una terza $\left(X^{\prime \prime}, Y^{\prime \prime}, Z^{\prime \prime}\right)$, ortogonale ad entrambe.

Fra le tre direzioni sussisteranno, come è noto, le tre terne di relazioni :

(A)

$$
\left\{\begin{array}{c}
X=Y^{\prime} Z^{\prime \prime}-Z^{\prime} Y^{\prime \prime} \\
Y=Z^{\prime} X^{\prime \prime}-X^{\prime} Z^{\prime \prime} \\
Z=X^{\prime} Y^{\prime \prime}-Y^{\prime} X^{\prime \prime} \\
X^{\prime}=Y^{\prime \prime} Z-Z^{\prime \prime} Y \\
\ldots \ldots \ldots \ldots \\
\ldots \ldots \ldots \ldots \\
X^{\prime \prime}=Y Z^{\prime}-Z Y^{\prime} \\
\ldots \ldots \ldots \ldots \\
\ldots \ldots \ldots \ldots
\end{array}\right.
$$

Confrontando ora la prima e la seconda di queste terne, rispettivamente con le (14) e (14') si avra immediatamente
(г6) $\left\{\begin{array}{l}\frac{\partial X}{\partial \sigma}=X^{\prime \prime} \frac{D}{\lambda}, \\ \frac{\partial Y}{\partial \sigma}=Y^{\prime \prime} \frac{D}{\lambda}, \\ \frac{\partial Z}{\partial \sigma}=Z^{\prime \prime} \frac{D}{\lambda} .\end{array}\right.$

$$
\left(\text { I6) } \left\{\begin{array}{l}
\frac{\partial X^{\prime}}{\partial \sigma}=-X^{\prime \prime} \frac{D^{\prime}}{\lambda} \\
\frac{\partial Y^{\prime}}{\partial \sigma}=-Y^{\prime \prime} \frac{D^{\prime}}{\lambda} \\
\frac{\partial Z^{\prime}}{\partial \sigma}=-Z^{\prime \prime} \frac{D^{\prime}}{\lambda} .
\end{array}\right.\right.
$$

Siamo in grado, inoltre, di procurarci delle espressioni analoghe per le derivate parziali $\frac{\partial X}{\partial z}, \ldots$ e $\frac{\partial X^{\prime}}{\partial z}, \ldots$

Difatti, dal seguente sistema:

$$
\left\{\begin{array}{l}
\sum X \frac{\partial X}{\partial z}=0, \\
\sum X^{\prime} \frac{\partial X}{\partial z}=-\lambda, \\
\sum X^{\prime \prime} \frac{\partial X}{\partial z}=\frac{\lambda}{D} \sum \frac{\partial X}{\partial z} \frac{\partial X}{\partial \sigma},
\end{array}\right.
$$

di cui le due prime equazioni ci sono note, e la terza si ottiene imme- 
diatamente dalle (I6), otteniamo subito, tenendo eziandio presenti le $(A)$ :

$\left(16^{\prime \prime}\right)$

$$
\left\{\begin{array}{l}
\frac{\partial X}{\partial z}=-\lambda X^{\prime}+\frac{\lambda}{D} X^{\prime \prime} \sum \frac{\partial X}{\partial \sigma} \frac{\partial X}{\partial \tau}, \\
\frac{\partial Y}{\partial z}=-\lambda Y^{\prime}+\frac{\lambda}{D} Y^{\prime \prime} \sum \frac{\partial X}{\partial \sigma} \frac{\partial X}{\partial z} \\
\frac{\partial Z}{\partial z}=-\lambda Z^{\prime}+\frac{\lambda}{D} Z^{\prime \prime} \sum \frac{\partial X}{\partial \sigma} \frac{\partial X}{\partial z} .
\end{array}\right.
$$

In modo perfettamente analogo, e considerando il sistema:

(I8)

$$
\left\{\begin{array}{l}
\sum X \frac{\partial X^{\prime}}{\partial z}=\lambda, \\
\sum X^{\prime} \frac{\partial X^{\prime}}{\partial z}=0, \\
\sum X^{\prime \prime} \frac{\partial X^{\prime}}{\partial z}=-\frac{\lambda}{D^{\prime}} \sum \frac{\partial X^{\prime}}{\partial z} \frac{\partial X^{\prime}}{\partial \sigma},
\end{array}\right.
$$

si deducono i valori:

$\left(16^{\prime \prime \prime}\right)$

$$
\left\{\begin{array}{l}
\frac{\partial X^{\prime}}{\partial z}=\lambda X-\frac{\lambda}{D^{\prime}} X^{\prime \prime} \sum \frac{\partial X^{\prime}}{\partial z} \frac{\partial X^{\prime}}{\partial \sigma}, \\
\frac{\partial Y^{\prime}}{\partial z}=\lambda Y-\frac{\lambda}{D^{\prime}} Y^{\prime \prime} \sum \frac{\partial X^{\prime}}{\partial z} \frac{\partial X^{\prime}}{\partial \sigma}, \\
\frac{\partial Z^{\prime}}{\partial z}=\lambda Z-\frac{\lambda}{D^{\prime}} Z^{\prime \prime} \sum \frac{\partial X^{\prime}}{\partial z} \frac{\partial X^{\prime}}{\partial \sigma} .
\end{array}\right.
$$

Riprendiamo ora le equazioni (9') e sostituiamo in esse $i$ valori (16), $\left(16^{\prime}\right),\left(16^{\prime \prime}\right),\left(16^{\prime \prime \prime}\right)$; otterremo:

$$
(19)\left\{\begin{array}{c}
\lambda \beta X+a \frac{D}{\lambda} X^{\prime \prime}-\alpha\left(-\lambda X^{\prime}+\frac{\lambda}{D} X^{\prime \prime} \sum \frac{\partial X}{\partial \sigma} \frac{\partial X}{\partial z}\right) \\
-\lambda \alpha X^{\prime}-b \frac{D^{\prime}}{\lambda} X^{\prime \prime}-\beta\left(\lambda X-\frac{\lambda}{D^{\prime}} X^{\prime \prime} \sum \frac{\partial X^{\prime}}{\partial z} \frac{\partial X^{\prime}}{\partial \sigma}\right)=0, \\
-\lambda \alpha Y^{\prime}-b \frac{D^{\prime}}{\lambda} Y^{\prime \prime}-\beta\left(\lambda Y-\frac{\lambda}{D^{\prime}} Y^{\prime \prime} \sum \frac{\partial X^{\prime}}{\partial z} \frac{\partial X^{\prime}}{\partial \sigma}\right)=0, \\
\lambda \beta Z+a \frac{D}{\lambda} Z^{\prime \prime}-\alpha\left(-\lambda Z^{\prime}+\frac{\lambda}{D} Z^{\prime \prime} \sum \frac{\partial X}{\partial \sigma} \frac{\partial X}{\partial z}\right) \\
-\lambda \alpha Z^{\prime}-b \frac{D^{\prime}}{\lambda} Z^{\prime \prime}-\beta\left(\lambda Z-\frac{\lambda}{D^{\prime}} Z^{\prime \prime} \sum \frac{\partial X^{\prime}}{\partial z} \frac{\partial X^{\prime}}{\partial \sigma}\right)=0 ;
\end{array}\right.
$$

e da queste, moltiplicando li prima per $X^{\prime \prime}$, la seconda per $Y^{\prime \prime}$, la terza 
per $Z^{\prime \prime}$ e sommando, deduciamo l'equazione:

(20) $a \frac{D}{\lambda}-\alpha \frac{\lambda}{D} \sum \frac{\partial X}{\partial \sigma} \frac{\partial X}{\partial z}-b \frac{D^{\prime}}{\lambda}+\beta \frac{\lambda}{D^{\prime}} \sum \frac{\partial X^{\prime}}{\partial z} \frac{\partial X^{\prime}}{\partial \sigma}=0$.

Non è difficile riconoscere come da questa equazione dipende interamente la risoluzione del problema che ci siamo proposti.

Tale risoluzione, intanto, noi l'abbiamo fatta apparire dipendente dalle sei incognite $X, Y, Z ; X^{\prime}, Y^{\prime}, Z^{\prime}$.

È facile vedere come $X^{\prime}, Y^{\prime}, Z^{\prime}$ si esprimano facilmente per $X, Y, Z$ : quanto poi a queste dimostreremo dopo come esse si possano ritenere interamente arbitrarie, e, quindi, si possano supporre sempre date, pur di soddisfare alla relazione $X^{2}+Y^{2}+Z^{2}=\mathrm{r}$.

Formiamoci, innanzitutto, il $\sum d X^{2}$.

Avremo facilmente, utilizzando $i$ valori gid trovati per $\frac{\partial X}{\partial z}$, etc.:

$$
\left\{\begin{aligned}
d X^{2}+d Y^{2}+d Z^{2}=\left[\lambda^{2}+\right. & \left.\frac{\lambda^{2}}{D^{2}}\left(\sum \frac{\partial X}{\partial z} \frac{\partial X}{\partial \sigma}\right)^{2}\right] d z^{2} \\
& +2 \sum \frac{\partial X}{\partial \sigma} \frac{\partial X}{\partial z} d z d \sigma+\frac{D^{2}}{\lambda^{2}} d \sigma^{2} ;
\end{aligned}\right.
$$

e se poniamo per brevita:

$$
\left\{\begin{array} { c } 
{ \frac { \lambda } { D } \sum \frac { \partial X } { \partial \sigma } \frac { \partial X } { \partial z } = - q , } \\
{ \frac { D } { \lambda } = - q _ { 1 } , }
\end{array} \left\{\begin{array}{c}
\frac{\lambda}{D^{\prime}} \sum \frac{\partial X^{\prime}}{\partial z} \frac{\partial X^{\prime}}{\partial \sigma}=p \\
-\frac{D^{\prime}}{\lambda}=p_{1},
\end{array}\right.\right.
$$

sarà allora:

(23) $d X^{2}+d Y^{2}+d Z^{2}=\left(\lambda^{2}+q^{2}\right) d z^{2}+2 q q_{\mathrm{I}} d z d \sigma+q_{\mathrm{I}}^{2} d \sigma^{2}$.

Formiamo su quest'espressione il $\Delta(\chi, X)$; avremo subito:

$$
\Delta(z, X)=-\frac{\mathrm{I}}{\lambda^{2}} \frac{q}{q_{\mathrm{x}}} \frac{\partial X}{\partial \sigma}+\frac{\mathrm{I}}{\lambda^{2}} \frac{\partial X}{\partial z},
$$

donde, sostituendo a $\frac{\partial X}{\partial z}$ e $\frac{\partial X}{\partial \sigma}$ i valori già visti

$$
\begin{gathered}
\frac{\partial X}{\partial z}=-\lambda X^{\prime}+\frac{\lambda}{D} X^{\prime \prime} \sum \frac{\partial X}{\partial \sigma} \frac{\partial X}{\partial z}, \\
\frac{\partial X}{\partial \sigma}=X^{\prime \prime} \frac{D}{\lambda},
\end{gathered}
$$


avremo

$\mathrm{e}$ in modo analogo:

$$
X^{\prime}=-\frac{\Delta(z, X)}{\lambda}
$$

$$
\begin{aligned}
& Y^{\prime}=-\frac{\Delta(z, Y)}{\lambda}, \\
& Z^{\prime}=-\frac{\Delta(z, Z)}{\lambda} .
\end{aligned}
$$

Ma è facile avere il valore di $\lambda$ in funzione di $z$. Difatti, dal sistema (14) si trae facilmente:

da cui

$$
I=\frac{\lambda^{2}}{D^{2}}\left[\left(\frac{\partial X}{\partial \sigma}\right)^{2}+\left(\frac{\partial Y}{\partial \sigma}\right)^{2}+\left(\frac{\partial Z}{\partial \sigma}\right)^{2}\right],
$$

$$
\frac{\mathrm{I}}{\lambda^{2}}=\frac{\left(\frac{\partial X}{\partial \sigma}\right)^{2}+\left(\frac{\partial Y}{\partial \sigma}\right)^{2}+\left(\frac{\partial Z}{\partial \sigma}\right)^{2}}{\left|\begin{array}{ccc}
\frac{\partial X}{\partial z} & \frac{\partial Y}{\partial z} & \frac{\partial Z}{\partial z} \\
\frac{\partial X}{\partial \sigma} & \frac{\partial Y}{\partial \sigma} & \frac{\partial Z}{\partial \sigma} \\
X & Y & Z
\end{array}\right|} .
$$

Tale valore di $\frac{\mathrm{I}}{\lambda^{2}}$ non è altro che il parametro differenziale primo $\Delta(z)$ rispetto alla forma dell'elemento lineare nella rappresentazione sferica

$$
d X^{2}+d Y^{2}+d Z^{2}=e_{11} d z^{2}+2 e_{\mathrm{r} 2} d z d \sigma+e_{22} d \sigma^{2} .
$$

Dunque

$$
\frac{\mathrm{I}}{\lambda^{2}}=\Delta(z)
$$

e le formole che dànno $X^{\prime}, Y^{\prime}, Z^{\prime}$ si potranno scrivere:

$$
\left\{\begin{array}{l}
X^{\prime}=-\frac{\Delta(z, X)}{\sqrt{\Delta(z)}}, \\
Y^{\prime}=-\frac{\Delta(z, Y)}{\sqrt{\Delta(z)}}, \\
Z^{\prime}=-\frac{\Delta(z, Z)}{\sqrt{\Delta(z)}} .
\end{array}\right.
$$

Infine, sostituendo tali lavori nelle equazioni (4) esse diverranno: 


$$
\left\{\begin{array}{l}
d \xi=\left[a X-b \frac{\Delta(z, X)}{\sqrt{\Delta(z)}}\right] d z+\left[\alpha X-\beta \frac{\Delta(z, X)}{\sqrt{\Delta(z)}}\right] d \sigma, \\
d n=\left[a Y-b \frac{\Delta(z, Y)}{\sqrt{\Delta(z)}}\right] d z+\left[\alpha Y-\beta \frac{\Delta(z, Y)}{\sqrt{\Delta(z)}}\right] d \sigma, \\
d \zeta=\left[a Z-b \frac{\Delta(z, Z)}{\sqrt{\Delta(z)}}\right] d z+\left[\alpha Z-\beta \frac{\Delta(z, Z)}{\sqrt{\Delta(z)}}\right] d \sigma .
\end{array}\right.
$$

Ora noi ci eravamo proposti di determ nare $X, Y, Z ; X^{\prime}, Y^{\prime}, Z^{\prime}$ in guisa tale che le espressioni $d i d \xi, d n, d \zeta$ risultassero differenziali esatti.

Tali valori di $X, Y, Z ; X^{\prime}, Y^{\prime}, Z^{\prime}$ sarebbero stati quelli ottenuti dal sistema (9). Le $X^{\prime} Y^{\prime} Z^{\prime}$ abbiamo visto che per le (25) ci risultano espresse per $X, Y, Z$; quanto a queste ultime, soddisfacendo esse alla relazione $X^{2}+Y^{2}+Z^{2}=\mathrm{I}$ sono le coordinate di un punto di una superficie sferica di raggio $I$; le loro espressioni in funzione di un sistena di coordinate curvilinee $u, v$ scelte sulla sfera, varieranno col variar del sistema; tenendo questo arbitrario, potremo intendere date ad arbitrio le espressioni di $X, Y, Z$ in funzione di quelle coordinate curvilinee $u, v$, purchè, però, $X, Y, Z$ siano sempre le coordinate di un punto della superficie sferrica, ossia soddisfino sempre alla relazione:

$$
X^{2}+Y^{2}+Z^{2}=\mathrm{I} \text {. }
$$

Ma, sebbene appaia, dunque, lecito il tenere $X, Y, Z$ arbitrarie, nulla dice che le espressioni (25) siano differenziali esatti, quantunque ad $X^{\prime}, Y^{\prime}, Z^{\prime}$ si sinno sostituiti valori in funzione di $X, Y, Z$, dedotte dalle equazioni del sistema (9).

Per altro osserviamo che noi, operando interamente su quel sistema abbiamo dedotto l'equazione:

$$
a \frac{D}{\lambda}-\alpha \frac{\lambda}{D} \sum \frac{\partial X}{\partial \sigma} \frac{\partial X}{\partial z}-b \frac{D^{\prime}}{\lambda}+\beta \frac{\lambda}{D^{\prime}} \sum \frac{\partial X^{\prime}}{\partial z} \frac{\partial X^{\prime}}{\partial \sigma}=0
$$

che per le abbreviazioni introdotte si potrà scrivere:

$$
-a q_{\mathrm{I}}+\alpha q+b p_{\mathrm{I}}-\beta p=0 .
$$

Se in questa equazione riusciremo ad esprimere $p, q, p_{\mathrm{s}}$ e $q_{\mathrm{s}}$ per $z$ e $\sigma$, e se potremo trovare, o stabilire, una relazione che dia p. es. la $\sigma$ in funzione della $z$, avremio una equazione che conterrd la sola $z$.

Supponiamo, per di più, di potere esprimere la $z$ di quest'equazione in funzione di $u$ e $v$; allora è chinro che ogni integrale di essa 
equazione, sostituito nelle espressioni (25), renderà $d \xi, d n, d \zeta$ differenziali esatti.

Tutto sta a vedere come potremo esprimere $p, q$, etc. in funzione di $z$ e $\sigma$, e la $z$ in funzione di sola $u$ e $v$.

Evidentemente, basterì per questo prendere la forma:

(23) $d X^{2}+d Y^{2}+d Z^{2}=\left(\lambda^{2}+q^{2}\right) d z^{2}+2 q q_{1} d z d \sigma+q_{1}^{2} d \sigma^{2}$,

che per l'introduzione del sistema arbitrario $u, v$ potremo porre:

$$
=e_{11} d u^{2}+2 e_{12} d u d v+e_{22} d v^{2},
$$

essendo $e_{11}, e_{12}, e_{22}$ note funzioni di $X, Y, Z$.

Quindi cercheremo esprimere $p, q$, etc. per forme invariantive, fatte sulla (23), ove entrino solo $z$ e $\sigma$, e che, essendo invariantive, potranno ancor intendersi fatte sulla $\left(23^{\prime}\right)$, si che $z$ e $\sigma$ appariranno funzioni di $u$ e $v$.

Infine, riuscendo ad esprimere $\sigma$ per $z$, e sostituendo nell'equazione (20) non avremo che ad integrare l'equazione cosi ottenuta, ogni integrale $z$ funzione di $u$ e $v$ di quell'equazione rendendo, per quanto precede, differenziali esatti le espressioni (25) di $d \xi, d n, d \zeta$.

In sostanza, dato allora il quadrato dell'elemento lineare di una superficie $S$ :

$$
\left(a^{2}+b^{2}\right) d z^{2}+2(a \alpha+b \beta) d z d \sigma+\left(\alpha^{2}+\beta^{2}\right) d \sigma^{2},
$$

dove $a, b, \alpha, \beta$ sono le funzioni di $z e \sigma$ definite nel modo visto in principio, e scelte le quantita $X, Y, Z$ sono legate tra di loro dalla relazione:

$$
X^{2}+Y^{2}+Z^{2}=I
$$

e, per conseguenza, coordinate dei punti di una superficie sferica di raggio $I$, il cui elemento lineare sarà dato $d a$

$$
d X^{2}+d Y^{2}+d Z^{2}=e_{11} d u^{2}+2 e_{12} d u d v+e_{22} d v^{2},
$$

allora ogni funzione $z$ di $u, v$, integrale dell'equazione differenziale (20)

$$
-a q_{\mathrm{r}}+\alpha q+b p_{\mathrm{r}}-\beta p=0,
$$

renderà differenziali esatti le espressioni di $d \xi, d n, d \zeta$ e darà, p. c., tutte le superficie applicabili sulla superficie data.

Vedremo più in là in qual modo si possa, e convenga, esprimere 
$\sigma$ per $z$, e come ciò limiti la generalità delle coordinate $z$ e $\sigma$, a cui va riferita la superficie $S$.

Cerchiamo, dunque, di esprimere $p, q, p_{1}, q_{\mathrm{r}}$ per $z$ e $\sigma$ sotto forma invariantiva.

Innanzitutto, procuriamoci delle relazioni tra $p, q, p_{\mathrm{I}}, q_{\mathrm{I}}$, relazioni che, come fra poco vedremo, ci saranno utili.

Consideriamo i due sistemi seguenti:

$$
\begin{array}{ll}
\sum X \frac{\partial X^{\prime \prime}}{\partial z}=q, & \sum X \frac{\partial X^{\prime \prime}}{\partial \sigma}=q_{1}, \\
\sum X^{\prime} \frac{\partial X^{\prime \prime}}{\partial z}=-p, & \sum X^{\prime} \frac{\partial X^{\prime \prime}}{\partial \sigma}=-p_{1}, \\
\sum X^{\prime \prime} \frac{\partial X^{\prime \prime}}{\partial z}=0, & \sum X^{\prime \prime} \frac{\partial X^{\prime \prime}}{\partial \sigma}=0,
\end{array}
$$

la cui formazione è evidente, tenuto conto delle formole che precedono.

$\mathrm{Da}$ tali sistemi si traggono i valori

$(\alpha)\left\{\begin{array}{l}\frac{\partial X^{\prime \prime}}{\partial z}=q X-p X^{\prime} \\ \frac{\partial Y^{\prime \prime}}{\partial z}=q Y-p Y^{\prime} \\ \frac{\partial Z^{\prime \prime}}{\partial z}=q Z-p Z^{\prime}\end{array} \quad(\beta) \quad\left\{\begin{array}{l}\frac{\partial X^{\prime \prime}}{\partial \sigma}=q_{\mathrm{s}} X-p_{\mathrm{I}} X^{\prime} \\ \frac{\partial Y^{\prime \prime}}{\partial \sigma}=q_{\mathrm{I}} Y-p_{\mathrm{I}} Y^{\prime} \\ \frac{\partial Z^{\prime \prime}}{\partial \sigma}=q_{\mathrm{I}} Z-p_{\mathrm{x}} Z^{\prime} .\end{array}\right.\right.$

Scriviamo ora coi simboli $p, q, p_{1}, q_{1}$ le espressioni (I6), (16'), ( $\left.16^{\prime \prime}\right)$ e $\left(I 6^{\prime \prime \prime}\right)$; da esse ricaviamo subito:

(m)

$$
\left\{\begin{array}{l}
d X=\left(-\lambda X^{\prime}-q X^{\prime \prime}\right) d z-q_{x} X^{\prime \prime} d \sigma \\
d Y=\ldots \ldots \ldots \\
d Z=\ldots \ldots \ldots \\
d X^{\prime}=\left(\lambda X+\ldots_{\ldots}^{\prime \prime}\right) d z+p_{1} X^{\prime \prime} d \sigma \\
d Y^{\prime}=\ldots \ldots \ldots \\
d Z^{\prime}=\ldots \ldots \ldots
\end{array}\right.
$$

donde, scrivendo le condizioni d'integrabilita: 


$$
\begin{aligned}
& \frac{\partial\left(\lambda X^{\prime}+q X^{\prime \prime}\right)}{\partial \sigma}=\frac{\partial\left(q_{1}, X^{\prime \prime}\right)}{\partial z} \\
& \frac{\partial\left(\lambda X+p X^{\prime \prime}\right)}{\partial \sigma}=\frac{\partial\left(p_{1}, X^{\prime \prime}\right)}{\partial z}
\end{aligned}
$$

si hanno le due terne:

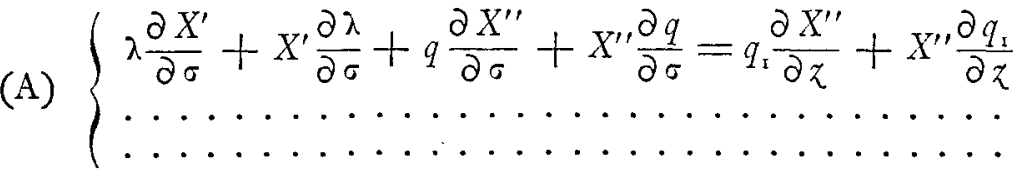



Dalla terna (A), moltiplicando per $X^{\prime \prime}, Y^{\prime \prime}, Z^{\prime \prime}$ e sommando, si trae subito la relazione:

$(26)^{\prime}$

$$
\frac{\partial q}{\partial \sigma}-\frac{\partial q_{\mathrm{r}}}{\partial z}=-\lambda p_{\mathrm{x}}
$$

Analogamente, dalla terna (B) si trae l'altra:

$$
\frac{\partial p}{\partial \sigma}-\frac{\partial p_{r}}{\partial z}=\lambda q_{x}
$$

Infine, ancor dalla prima terna, ma moltiplicando per $X^{\prime}, Y^{\prime}, Z^{\prime}$ e sommando si trae, tenendo presenti le $(x)$ e $(\beta)$, $(26)^{\prime \prime \prime}$

$$
p q_{\mathrm{r}}-q p_{\mathrm{r}}=-\frac{\partial \lambda}{\partial \sigma} \text {. }
$$

Prendiamo ora la (23)

(23) $d X^{2}+d Y^{2}+d Z^{2}=\left(\lambda^{2}+q^{2}\right) d z^{2}+2 q q_{\mathrm{I}} d z d \sigma+q_{\mathrm{x}}^{2} d \sigma^{2}$

e formiamo su questa il parametro differenziale misto $\Delta\left(z, \frac{I}{\lambda^{2}}\right)$.

Avremo facilmente :

$$
\Delta\left(z, \frac{I}{\lambda^{2}}\right)=\frac{-\frac{q}{q_{1}} \frac{\partial \frac{\mathrm{I}}{\lambda^{2}}}{\partial \sigma}+\frac{\partial \frac{\mathrm{I}}{\lambda^{2}}}{\partial \tau}}{\lambda^{2}},
$$


da cui

(27)

$$
\frac{q}{q_{1}}=\frac{\frac{\partial \frac{\mathrm{I}}{\lambda^{2}}}{\partial z}-\lambda^{2} \Delta\left(z, \frac{\mathbf{I}}{\lambda^{2}}\right)}{\frac{\partial \frac{\mathrm{I}}{\lambda^{2}}}{\partial \sigma}} .
$$

Formiamoci ora il $\Delta_{2}(z)$.

Eseguendo i calcoli, e dopo qualche semplificazione, si ottiene:

$$
\Delta_{2}(z)=-\frac{I}{\lambda^{2} q_{1}}\left(\frac{\partial q}{\partial \sigma}-\frac{\partial q_{1}}{\partial z}\right)+\frac{I}{\lambda^{3}}\left(\frac{q}{q_{1}} \frac{\partial \lambda}{\partial \sigma}-\frac{\partial \lambda}{\partial z}\right),
$$

donde per la $(26)^{\prime}$ e per la (27):

$$
\Delta^{2}(z)=\frac{I}{\lambda} \frac{p_{I}}{q_{1}}+\frac{\lambda^{2}}{2} \Delta\left(z, \frac{I}{\lambda^{2}}\right) .
$$

Dalla quale, in fine, si ricava:

$$
\frac{p_{1}}{q_{I}}=\lambda \Delta_{2}(z)-\frac{\lambda^{3}}{2} \Delta\left(z, \frac{I}{\lambda^{2}}\right) \text {. }
$$

Calcoliamoci ora, sempre sulla forma (23) dell'elemento lineare, linvariante $\sigma(u)$ del Darboux, espresso dalla formola:

ossia per noi:

$$
\sigma(u)=\frac{\Delta \Delta(u)-2 \Delta_{2}(u) \Delta[u, \Delta(u)]}{4 \Delta(u)},
$$

$$
\sigma(z)=\frac{\Delta \Delta(z)-2 \Delta_{2}(z) \Delta[z, \Delta(z)]}{4 \Delta(z)}
$$

Ricordando che è $\Delta(z)=\frac{\mathrm{I}}{\lambda^{2}}$ e che si è trovato

$$
\Delta_{2}(z)=\frac{I}{\lambda} \frac{p_{1}}{q_{1}}+\frac{\lambda^{2}}{2} \Delta\left(z, \frac{I}{\lambda^{2}}\right),
$$

dopo breve calcolo si ottiene:

$$
\sigma(z)=\frac{\lambda^{2}\left(\frac{\partial \frac{I}{\lambda^{2}}}{\partial \sigma}\right)^{2}+2 \frac{I}{\lambda} p_{1} q \frac{\partial \frac{I}{\lambda^{2}}}{\partial \sigma}-2 \frac{I}{\lambda} p_{\mathrm{I}} q_{\mathrm{I}} \frac{\partial \frac{\mathrm{I}}{\lambda^{2}}}{\partial z}}{4 q_{\mathrm{I}}^{2}}
$$

Indichiamo pure con $\theta(i)$, come $f_{a}$ il WeIngarten, questo inva- 
riante; dall'ultima formola si ha facilmente:

$$
0(z)=\frac{\mathrm{I}}{\lambda^{4}} \frac{\mathrm{I}}{q_{\mathrm{I}}^{2}}\left(\frac{\partial \lambda}{\partial \sigma}\right)^{2}-\frac{\mathrm{I}}{2 \lambda} \frac{p_{\mathrm{I}}}{q_{\mathrm{I}}} \lambda^{2} \Delta\left(z, \frac{\mathrm{I}}{\lambda^{2}}\right),
$$

ossia, applicando la $(26)^{\prime \prime \prime}$,

$$
\theta(z)=\frac{\mathrm{I}}{\lambda^{4}} \frac{p_{\mathrm{x}}}{q_{\mathrm{I}}} \frac{\partial \lambda}{\partial \sigma}-\frac{\mathrm{I}}{\lambda^{4}} \frac{q}{q_{1}} \frac{p_{\mathrm{I}}}{q_{\mathrm{I}}} \frac{\partial \lambda}{\partial \sigma}-\frac{\lambda}{2} \Delta\left(z, \frac{\mathrm{I}}{\lambda^{2}}\right)\left[\lambda \Delta_{2}(z)-\frac{\lambda^{3}}{2} \Delta\left(z, \frac{\mathrm{I}}{\lambda^{2}}\right)\right],
$$

da cui

$$
\text { (29) } \frac{p}{q_{\mathrm{I}}}=\frac{\mathrm{I}}{\frac{\mathrm{I}}{\lambda^{4}} \frac{\partial \lambda}{\partial \sigma}}\left\{\theta(z)+\frac{\mathrm{I}}{\lambda^{4}} \frac{\partial \lambda}{\partial \sigma} \frac{\frac{\partial \frac{\mathrm{I}}{\lambda^{2}}}{\partial z}-\lambda^{2} \Delta\left(z, \frac{\mathrm{I}}{\lambda^{2}}\right)}{\frac{\partial \frac{\mathrm{I}}{\lambda^{2}}}{\partial \sigma}}\left[\lambda \Delta_{2}(z)-\frac{\lambda^{3}}{2} \Delta\left(z, \frac{\mathrm{I}}{\lambda^{2}}\right)\right] .\right.
$$

I parametri differenziali che figurano nelle espressioni (27), (28), (29) s'intendono fatti sulla forma dell'elemento lineare nella rappresentazione sferica particolare:

(23) $d X^{2}+d Y^{2}+d Z^{2}=\left(\lambda^{2}+q^{2}\right) d z^{2}+2 q q_{\mathrm{I}} d z d \sigma+q_{\mathrm{I}}^{2} d \sigma^{2}$.

$\mathrm{Ma}$, essendo i parametri differenziali forme invariantive, essi possono anche intendersi fatti sulla forma seguente:

$$
d X^{2}+d Y^{2}+d Z^{2}=e_{11} d u^{2}+2 e_{12} d u d v+e_{22} d v^{2},
$$

ossia le espressioni di $\frac{q}{q_{\mathrm{I}}}, \frac{p_{\mathrm{I}}}{q_{\mathrm{r}}}, \frac{p}{q_{\mathrm{r}}}$ date dalle (27), (28) e (29) possono intendersi funzioni delle variabili $z$ e $\sigma$, di cui, quest'ultima, funzione di $u$ e $v$.

Prima di sostituire gli anzidetti valori di $\frac{q}{q_{\mathrm{x}}}, \frac{p_{\mathrm{I}}}{q_{\mathrm{s}}}, \frac{p}{q_{\mathrm{x}}}$ nell'equazione (20)

$$
-a q_{\mathrm{I}}+b p_{\mathrm{s}}+\alpha q-\beta p=0
$$

dopo averla divisa per $q_{\text {s }}$ che non si annulla, notiamo che quei valori 
si semplificano moltissimo facendo

$$
\frac{\mathrm{I}}{\lambda^{2}}=\sigma=\Delta(z)
$$

Essi divengono, infatti:

$$
\begin{gathered}
\frac{q}{q_{\mathrm{I}}}=-\frac{\mathrm{I}}{\sigma} \Delta(z, \sigma), \\
\frac{p_{\mathrm{I}}}{q_{\mathrm{I}}}=\frac{\mathrm{I}}{\sqrt{\sigma}} \Delta_{2}(z)-\frac{\mathrm{I}}{2 \sqrt{\sigma^{3}}} \Delta(z, \sigma), \\
\frac{p}{q_{\mathrm{I}}}=-\frac{2}{\sqrt{\sigma}} \theta(z) .
\end{gathered}
$$

Ora, porre $\sigma=\frac{\mathrm{I}}{\lambda^{2}}$ equivale operare un cambiamento dei parametri ai quali si riferisce la superficie data, ossia, anzicchè intendere la superficie riferita alle coordinate $z=$ cost., $\sigma=$ cost., intenderla riferita alle coordinate

$$
z_{1}=z=\text { cost. }, \quad \sigma_{1}=\frac{1}{\lambda^{2}}=\text { cost. },
$$

si che l'elemento sia

$$
E_{\mathrm{r}} d z^{2}+2 F_{\mathrm{x}} d z d \sigma_{\mathrm{z}}+G_{\mathrm{I}} d \sigma_{\mathrm{r}}^{2} .
$$

Di qui appare l'utilità dell'artifizio del WeIngarten, di ricorrere alla forma ( $R$ ), che non è se non che la sua forma ridotta.

Sostituendo allora i valori $\left(27^{\prime}\right),\left(28^{\prime}\right),\left(29^{\prime}\right)$ nella (20) essa diviene:

$$
(20)^{\prime}-a+b\left[\frac{\mathrm{I}}{\sqrt{\sigma}} \Delta_{2}(z)-\frac{\mathrm{I}}{2 \sqrt{\sigma^{3}}} \Delta(z, \sigma)\right]-\alpha \frac{\mathrm{I}}{\sigma} \Delta(z, \sigma)+\beta \frac{2}{\sqrt{\sigma}} \theta(z)=0 .
$$

$E$ però, riprendendo quanto già si accennò a pagina 82 , potremo dire che:

Dato l'elemento lineare di una superficie $S$ :

$$
d s=\sqrt{\left(a^{2}+b^{2}\right) d z^{2}+2(a \alpha+b \beta) d z d \sigma+\left(\alpha^{2}+\beta^{2}\right) d \sigma^{2}},
$$

dove $a, b, \alpha, \beta$ sono le funzioni di $z$ e $\sigma$ definite nel modo già visto in principio per mezzo di $E, F, G$ COEFFICIENTI DI UNA FORMA RIDOTTA DEL Weingarten, e scelte le funzioni $X, Y, Z$ solo legate tra di loro dalla relazione $X^{2}+Y^{2}+Z^{2}=\mathrm{I} e$, p. c., coordinate dei punti di una superficie sferica di raggio $I$, il cui elemento lineare sarà dato da

$$
d X^{2}+d Y^{2}+d Z^{2}=e_{11} d u^{2}+2 e_{12} d u d v+e_{22} d v^{2}
$$


allora ogni funzione $z$ di $u$ e $v$, integrale dell'equazione differenziale

$$
\begin{aligned}
-a+b\left\{\frac{1}{\sqrt{\Delta(z)}} \Delta_{z}(z)-\right. & \left.\frac{1}{2 \sqrt{\Delta(z)^{3}}} \Delta[z, \Delta(z)]\right\} \\
& -\alpha \frac{1}{\Delta(z)} \Delta[z, \Delta(z)]+\beta \frac{2}{\sqrt{\Delta(z)}} \theta(z)=0
\end{aligned}
$$

renderà differenziali esatti le espressioni (25) di $d \xi, d n, d \zeta$, e darà, p. c., tutte le superficie applicabili sulla superficie data.

$\dot{E}$ facile dimostrare che l'equazione (20)' non è se non che l'equazione fondamentale (I7) del Weingarten:

$$
\text { (I7) } \quad a \alpha+2 a \sigma \Delta_{2}(z)-\frac{b+2 \alpha \sqrt{\sigma}}{\sqrt{\sigma}} J(z)-2 \beta \sqrt{\sigma} \theta(z)=0 \text {, }
$$

$o$, in altri termini, la coincidenza della nostra equazione

con l'equazione

$$
-a+b \frac{p_{\mathrm{r}}}{q_{1}}+\alpha \frac{q}{q_{1}}-\beta \frac{p}{q_{\mathrm{s}}}=0
$$

del Weingarten.

$$
-a+b \frac{P_{\mathrm{r}}}{Q_{\mathrm{T}}}+\alpha \frac{Q}{Q_{1}}-\beta \frac{P}{Q_{1}}=0
$$

Basterà, è chiaro, dimostrare l'identitì dei valori $\frac{p_{1}}{q_{1}}, \frac{q}{q_{1}}, \frac{p}{q_{1}}$ coi valori $\frac{P_{1}}{Q_{1}}, \frac{Q}{Q_{1}}, \frac{P}{Q_{1}}$.

Per questo, ricordiamo che trovammo le espressioni seguenti:

$$
\begin{aligned}
& \frac{q}{q_{\mathrm{i}}}=-\frac{\mathrm{I}}{\sigma} \Delta(z, \sigma), \\
& \frac{p_{1}}{q_{1}}=\frac{\mathrm{I}}{\sqrt{\sigma}} \Delta_{2}(z)-\frac{\mathrm{I}}{2 \sqrt{\sigma^{3}}} \Delta(z, \sigma), \\
& \frac{p}{q_{1}}=-\frac{2}{\sqrt{\sigma}} \theta(z) \text {. }
\end{aligned}
$$

Il Weingarten ottiene, invece,

$$
\left\{\begin{aligned}
\sigma \Delta_{2}(z)-J(z) & =-\frac{\mathrm{I}}{2} \sigma \frac{Q}{Q_{1}}, \\
J(z) & =-\sqrt{\sigma^{3}} \frac{P_{1}}{Q_{1}}, \\
\theta(z) & =-\frac{\sqrt{\sigma}}{2} \frac{P}{Q_{1}} .
\end{aligned}\right.
$$


Il $\theta(z)$ essendo, sia nelle une, che nelle altre espressioni, l'invariante del Darboux, ne risulterà, intanto, che il valore $\mathrm{di} \frac{p}{q_{\mathrm{r}}}$, dato dalla (29)', ed il valore di $\frac{P}{Q_{\mathrm{I}}}$ dato dalla terza delle (IS) saranno uguali.

Dimostriamo che ciò avviene del pari per i valori $\frac{q}{q_{\mathrm{r}}}$ e $\frac{Q}{Q_{1}}$.

Se ciò è realmente, dovrà sussistere l'uguaglianza:

$$
-\frac{\mathbf{I}}{\sigma} \cdot \Delta(z, \sigma)=\frac{-\sigma \Delta_{2}(z)+J(z)}{\frac{\mathrm{I}}{2} \sigma} .
$$

Formiamoci, innanzitutto, linvariante $J(z)$ del Weingarten.

Esso ha l'espressione seguente:

$$
J(z)=\frac{\left(\frac{\partial z}{\partial u}\right)^{2} z_{22}-2 \frac{\partial z}{\partial u} \frac{\partial z}{\partial v} z_{12}+\left(\frac{\partial z}{\partial v}\right)^{2} z_{11}}{e_{11} e_{22}-e_{12}^{2}},
$$

se fatto sulla forma $\cdot e_{11} d u^{2}+2 e_{x 2} d u d v+e_{22} d v^{2}$.

Ma se lo facciamo sulla forma:

$\left(23^{\prime}\right) d X^{2}+d Y^{2}+d Z^{2}=\left(\frac{I}{\sigma}+q^{2}\right) d z^{2}+2 q q_{1} d z d \sigma+q_{1}^{2} d \sigma^{2}$

[che è la forma (23) ove si è posto $\left.\frac{I}{\lambda^{2}}=\sigma\right]$, esso diviene:

$$
J(z)=\frac{z_{22}}{\frac{\mathrm{I}}{\sigma} q_{\mathrm{x}}^{2}},
$$

dove $z_{22}$, che ha l'espressione generale seguente:

$$
z_{22}=\frac{\partial^{2} z}{\partial v^{2}}-\left(\begin{array}{c}
22 \\
1
\end{array}\right) \frac{\partial z}{\partial u}-\left(\begin{array}{c}
22 \\
2
\end{array}\right) \frac{\partial z}{\partial v}
$$

qui diviene $z_{22}=-\left(\begin{array}{c}2 \\ I\end{array}\right)$, essendo $\left(\begin{array}{c}22 \\ I\end{array}\right)$ il noto simbolo di CHrISTOFFEL, che andrà formato sulla $\left(23^{\prime}\right)$.

Risulterì, così:

$$
J(z)=\frac{-\left(\begin{array}{c}
22 \\
\mathrm{I}
\end{array}\right)}{\frac{\mathrm{I}}{\sigma} q_{\mathrm{I}}^{2}}=\frac{q q_{\mathrm{I}} \frac{\partial q_{\mathrm{I}}^{2}}{\partial \sigma}-2 q_{\mathrm{I}}^{2} \frac{\partial\left(q q_{\mathrm{I}}\right)}{\partial \sigma}+q_{\mathrm{I}}^{2} \frac{\partial q_{\mathrm{I}}^{2}}{\partial z}}{2\left(\frac{\mathrm{I}}{\sigma} q_{\mathrm{I}}^{2}\right)^{2}},
$$

Rend. Circ. Matem. Palermo, t. XVI (1902). - Stampato il 29 luglio rgor. 
da cui

$$
J(z)=\frac{\sigma^{2}}{q_{\mathrm{I}}} \frac{\partial q_{\mathrm{I}}}{\partial z}-\frac{\sigma^{2}}{q_{\mathrm{r}}} \frac{\partial q}{\partial \sigma}
$$

Ora formiamoci, sempre sulla $\left(23^{\prime}\right)$, il $\Delta(z, \sigma)$ ed il $\Delta_{z}(z)$. Si ottiene:

$$
\Delta(z, \sigma)=\frac{-q q_{\mathrm{r}}}{\frac{\mathrm{I}}{\sigma} q_{\mathrm{s}}^{2}}=-\sigma \frac{q}{q_{\mathrm{r}}},
$$

da cui

E per il $\Delta_{2}(z):$

$$
-\frac{\mathrm{I}}{\sigma} \Delta(z, \sigma)=\frac{q}{q_{\mathrm{I}}}
$$

$$
\Delta_{2}(z)=\frac{\sigma}{q_{\mathrm{s}}} \frac{\partial q_{\mathrm{I}}}{\partial z}-\frac{\sigma}{q_{\mathrm{I}}} \frac{\partial q}{\partial \sigma}-\frac{\mathrm{I}}{2} \frac{q}{q_{\mathrm{I}}} .
$$

Facendo allora le sostituzioni relative nella (3I), si ottiene da questa :

che è un'identità.

$$
\frac{q}{q_{1}}=\frac{q}{q_{1}}
$$

Si conclude, dunque, eziandlo l'uguaglianza dei due rapporti $\frac{q}{q_{1}}$ e $\frac{Q}{Q_{1}}$.

In modo analogo si dimostra essere $\frac{p_{\mathrm{r}}}{q_{\mathrm{r}}}=\frac{P_{\mathrm{I}}}{Q_{\mathrm{r}}}$.

Segue che, essendo i valori $\left(27^{\prime}\right),\left(28^{\prime}\right),\left(29^{\prime}\right)$ identici ai valori (IS) del Wiringarten, l'equazione fondan:entale (I7) di detto autore, non è se non che la nostra equazione $\left(20^{\prime}\right)$.

Il Weingarten pone, poi, la sua equazione fondamentale sotto la forma canonica, per la ricerca degli integrali col metodo delle caratteristiche.

Faremo ancora vedere come la nostra equazione (20)' si possa ridurre in modo diretto a tale forma ultima del Weingarten.

Come fa questo autore, adotteremo, per semplificare i calcoli, sulla sfera di raggio I le coordinate simmetriche.

Le coordinare $X, Y, Z$ di un punto qualunque della superficie di essa sfera saranno, com'è noto:

$$
X=\frac{x+y}{\mathrm{I}+x y}, \quad Y=\frac{i(y-x)}{\mathrm{I}+x y}, \quad Z=\frac{x y-\mathrm{I}}{\mathrm{I}+x y} .
$$


Il quadrato dell'elemento lineare sarà espresso dalla forma:

$$
d X^{2}+d Y^{2}+d Z^{2}=\frac{4 d x d y}{(I+x y)^{2}} .
$$

Adoperando le notazioni di Monge, avremo subito in questo caso le formole seguenti:

$$
\begin{gathered}
\sigma=\Delta(z)=(\mathrm{I}+x y)^{2} p q, \quad \Delta_{2}(\tilde{z})=(\mathrm{I}+x y)^{2} s, \\
\Delta(z, \sigma)=\Delta(z, \Delta z)=\frac{(\mathrm{I}+x y)^{4}}{2}\left\{2 p q s+p^{2}\left[t+\frac{2 q x}{\mathrm{I}+x y}+q^{2}\left(r+\frac{2 p y}{\mathrm{I}+x y}\right)\right]\right\}, \\
\theta(z)=\frac{(\mathrm{I}+x y)^{4}}{4}\left(r+\frac{2 p y}{\mathrm{I}+x y}\right)\left(t+\frac{2 q x}{\mathrm{I}+x y}\right)-\frac{(\mathrm{I}+x y)^{4}}{4} s^{2} .
\end{gathered}
$$

Sostituiamo tali valori nella nostra equazione $\left(20^{\prime}\right)$ :

$$
\left(20^{\circ}\right)-a+b\left[\frac{1}{\sqrt{\sigma}} \cdot \Delta_{2}(z)-\frac{1}{2 \sqrt{\sigma^{3}}} \Delta(z, \sigma)\right]-\alpha \frac{I}{\sigma} \Delta(z, \sigma)+\beta \frac{2}{\sqrt{\sigma}} \theta(z)=0 .
$$

Essa diverrà, svolgendo i calcoli,

$$
\begin{aligned}
& -4 a \sqrt{p^{3} q^{3}}+2 b s p q(\mathrm{I}+x y)-2 b p q s(\mathrm{I}+x y)-b p^{2} l(\mathrm{I}+x y) \\
& -2 b p^{2} q x-b q^{2} r(\mathrm{I}+x y)-2 b p q^{2} y-4 a \sqrt{p^{3} q^{3}} s(\mathrm{I}+x y)^{2}
\end{aligned}
$$$$
-2 p^{2} \sqrt{p q} \times t(\mathrm{I}+x y)^{2}-4 x x p^{2} q \sqrt{p q}(\mathrm{I}+x y)-2 \alpha q^{2} r \sqrt{p q}(\mathrm{I}+x y)^{2}
$$$$
-4 \alpha p q^{2} y(\mathrm{I}+x y) \sqrt{p q}-2 \beta r t p q(\mathrm{I}+x y)^{3}-4 \beta p^{2} q y t(\mathrm{I}+x y)^{2}
$$$$
-4 \beta p q^{2} r x(\mathrm{I}+x y)^{2}-8 \beta p^{2} q^{2} x y(\mathrm{I}+x y)-2 s^{2} \beta p q(\mathrm{I}+x y)^{3}=0 \text {. }
$$

In quest'equazione cambiamo segno; dividiamo per $2 \beta(\mathrm{I}+x y)^{3} p q$, poi aggiungiamo e togliamo l'espressione seguente:

$$
\begin{aligned}
\frac{b^{2} p^{2} q^{2}}{4 \beta^{2}(\mathrm{I}+x y)^{4} p^{2} q^{2}} & +\frac{4 x p^{2} b q^{2}(\mathrm{I}+x y) \sqrt{p q}}{4 \beta^{2}(\mathrm{r}+x y)^{4} p^{2} q^{2}}+\frac{4 \alpha^{2}(\mathrm{I}+x y)^{2} p^{3} q^{3}}{4 \beta^{2}(\mathrm{I}+x y)^{4} p^{2} q^{2}} \\
& +\frac{4 \alpha^{2} p q}{\beta^{2}(\mathrm{I}+x y)^{2}}+\frac{4 \alpha b \sqrt{p q}}{2 \beta^{2}(\mathrm{I}+x y)^{3}} .
\end{aligned}
$$

Fatto ciò, e scritti in un ordine opportuno $i$ varii termini, sarà facile mettere l'equazione sotto la forma seguente:

$$
\begin{gathered}
\left(r+\frac{2 y}{\mathrm{I}+x y} p+\frac{b+2 x(\mathrm{I}+x y) \sqrt{p q}}{2 \beta(\mathrm{I}+x y)^{2} p q} p^{2}\right)\left(t+\frac{2 x}{\mathrm{I}+x y} q+\frac{b+2 \alpha(\mathrm{I}+x y) \sqrt{p q}}{2 \beta(\mathrm{I}+x y)^{2} p q} q^{2}\right) \\
-\left[s-\frac{2 \frac{x}{\beta}(\mathrm{I}+x y) \sqrt{p q}}{(1+x y)^{2}}+\frac{b+2 \alpha(\mathrm{I}+x y) \sqrt{p q}}{2 \beta(\mathrm{I}+x y)^{2} p q} p q\right]^{2} \\
-\frac{4}{(\mathrm{I}+x y)^{3}} \frac{\alpha b-\beta a}{2 \beta^{2}}(\mathrm{I}+x y) \sqrt{p q}=0 .
\end{gathered}
$$


Da quest'equazione, poi, tenuto presente che in questo caso di coordinate simmetriche è

$$
\sigma=\Delta(z)=(1+x y)^{2} p q,
$$

e usando le notazioni seguenti del Werngartex

si passa all'altra:

$$
\lambda=\frac{\alpha}{\beta} \sqrt{\sigma}, \quad p=-\frac{b+2 \alpha \sqrt{\sigma}}{2 \beta \sigma},
$$

$$
\begin{gathered}
\left(r+\frac{2 y}{\mathrm{I}+x y} p-p p^{2}\right)\left(t+\frac{2 x}{\mathrm{I}+x y} q-p q^{2}\right)-\left(s-\frac{2 \lambda}{(\mathrm{I}+x y)^{2}}-p p q\right)^{2} \\
-\frac{4}{(\mathrm{I}+x y)^{4}} \frac{\alpha b-\beta a}{2 \beta^{2}} \sqrt{\sigma}=0,
\end{gathered}
$$

che è l'equazione che il Weingarten deduce dalla sua equazione fondamentale (I7).

$\mathrm{Da}$ questa equazione con le posizioni seguenti:

si passa subito all'altra:

$$
\begin{aligned}
W^{2} & =\frac{b \alpha-a \beta}{2 \beta^{2}} \sqrt{\sigma}, \\
\tau & =p \sigma+2(\lambda+i W), \\
\tau & =\rho \sigma+2(\lambda-i W),
\end{aligned}
$$

$$
\left(r+\frac{2 y}{\mathrm{I}+x y} p-p p^{2}\right)\left(t+\frac{2 x}{\mathrm{I}+x y} q-p q^{2}\right)-\left(s-\frac{\tau}{\sigma} p q\right)\left(s-\frac{\tau}{\sigma} p q\right)=0,
$$

che è della forma canonica

$$
(r-A)(t-B)-(s-C)(s-D)=0,
$$

$A, B, C, D$ essendo funzioni di $x, y, \tilde{z}, p, q$; e che meglio si presta alla ricerca degli integrali.

\section{Interpretawione geometrich dei risultati ottenuti.}

Abbiamo visto che in ogni punto $M$ di una superficie $S_{\mathrm{r}}$ applicabile sulla superficie data $S$, il quadrato del cui elemento lineare è espresso dalla forma:

$$
d s^{2}=\left(a^{2}+b^{2}\right) d z^{2}+2(a x+b \xi) d z d \sigma+\left(\alpha^{2}+\beta^{2}\right) d \sigma^{2},
$$

le direzioni $(X, Y, Z),\left(X^{\prime}, Y^{\prime}, Z^{\prime}\right),\left(X^{\prime \prime}, Y^{\prime \prime}, Z^{\prime \prime}\right)$ formano un triedro 
triretangolo, $X^{\prime \prime}, Y^{\prime \prime}, Z^{\prime \prime}$ essendo i coseni della normale in $M$ alla $S_{t}$, ed $X, Y, Z ; X^{\prime}, Y^{\prime}, Z^{\prime}$ i coseni di due direzioni tangenti alla stessa superficie.

Ma v'ha di più.

Calcoliamo l'angolo $\omega$ che p. es. la direzione $X, Y, Z$ forma con la linea coordinata $z=$ cost.

Ricordando i valori:

$$
\begin{aligned}
& X=\frac{\beta \frac{\partial \xi}{\partial z}-b \frac{\partial \xi}{\partial \sigma}}{\sqrt{\Delta}}, \\
& Y=\frac{\beta \frac{\partial n}{\partial z}-b \frac{\partial n}{\partial \sigma}}{\sqrt{\Delta}}, \\
& Z=\frac{\beta \frac{\partial \zeta}{\partial z}-b \frac{\partial \zeta}{\partial \sigma}}{\sqrt{\Delta}},
\end{aligned}
$$

e ricordando le note espressioni:

$$
\frac{1}{\sqrt{\alpha^{2}+\beta^{2}}} \frac{\partial \xi}{\partial \sigma}, \quad \frac{1}{\sqrt{\alpha^{2}+\beta^{2}}} \frac{\partial n}{\partial \sigma}, \quad \frac{1}{\sqrt{\alpha^{2}+\beta^{2}}} \frac{\partial \zeta}{\partial \sigma},
$$

dei coseni degli angoli che lin coordinata curvilinea $z=$ cost. passante per $M(\zeta, n, \zeta)$ fa con $\mathrm{i}$ tre assi coordinati, avremo subito:

$$
\cos \omega=\frac{1}{\sqrt{\alpha^{2}+\beta^{2}}} \frac{1}{\sqrt{a \beta-b \alpha}}\left[\beta(a \alpha+b \beta)-b\left(\alpha^{2}+\beta^{2}\right)\right]=f(a, b, \alpha, \beta) .
$$

Risulta, dunque, che l'angolo $\omega$ anzidetto è una funzione arbitraria deli'elemento lineare della superficie $S_{\mathrm{r}}$.

Tale funzione noi l'abbiamo definita in seguito, col porre tra $a, b, \alpha, \beta$ l'ulteriore relazione:

$$
\alpha\left(\frac{\partial a}{\partial \sigma}-\frac{\partial \alpha}{\partial z}\right)+\beta\left(\frac{\partial b}{\partial \sigma}-\frac{\partial \beta}{\partial z}\right)=0 .
$$

Consegue questo risultato importantissimo:

Per ogni deformazione che conservi l'elemento lineare della superficie il valore di quelliangolo rimane inalierato.

Il complesso delle direzioni come $(X, Y, Z),\left(X^{\prime}, Y^{\prime}, Z^{\prime}\right)$, costituisce una coppia di congruenze di rette, ortogonali tra di loro, e aventi per comune superficie iniziale la superficie considerata. 
Prendiamo in esame la congruenza formata dalle $(X, X, Z)$, quello che diremo per questa congruenza potendosi ripetere per l'altra $\left(X^{\prime}, Y^{\prime}, Z^{\prime}\right)$.

A definire la direzione $(X, Y, Z)$ concorreranno due parametri $u$ e $v$ che si dovranno intendere come un sistema qualunque di coordinate curvilinee, tracciate su di una sfera di raggio uguale all'unitá; conducendo per il centro di detta sfera una parallela alla direzione $(X, Y, Z)$, il punto ove tal parallela incontra la superficie sferica, rappresenterd per noi l'immagine della retta $(X, Y, Z)$ della congruenza, e sarà definito dalle sue coor Jinate curvilinee $(u, v)$ rispetto al sistema anzidetto.

Le due forme fondamentali della congruenza $(X, Y, Z)$ da noi considerata sono, com'è noto, le seguenti:

$$
\begin{aligned}
& \sum d X^{2}=E_{\mathrm{r}} d z^{2}+2 F_{\mathrm{s}} d z d \sigma+G_{\mathrm{x}} d \sigma^{2}, \\
& \sum d \xi d X=e d z^{2}+\left(f+f^{\prime}\right) d z d \sigma+g d \sigma^{2} .
\end{aligned}
$$

La $(\alpha)$ l'abbiamo gid trovata. Essa è :

$$
\sum d X^{2}=\left[\lambda^{2}+\frac{\lambda^{2}}{D^{2}}\left(\sum \frac{\partial X}{\partial z} \frac{\partial X}{\partial \sigma}\right)^{2}\right] d z^{2}+2 \sum \frac{\partial X}{\partial z} \frac{\partial X}{\partial \sigma} d z d \sigma+\frac{D^{2}}{\lambda^{2}} d \sigma^{2} .
$$

Formiamoci la ( $\beta$ ).

Avremo, adoperando i valori (4) ed i valori (16) e (16"):

$$
\begin{aligned}
\sum d_{\xi} d X & =\left[\left(a X+b X^{\prime}\right) d z+\left(\alpha X+\beta X^{\prime}\right) d \sigma\right]\left\{\left[-\lambda X^{\prime}+\frac{\lambda}{D} X^{\prime \prime} \sum \frac{\partial X}{\partial \sigma} \frac{\partial X}{\partial z}\right] d z+\frac{D}{\lambda} X^{\prime \prime} d \sigma\right\} \\
& +\left[\left(a Y+b Y^{\prime}\right) d z+\left(\alpha Y+\beta Y^{\prime}\right) d \sigma\right]\left\{\left[-\lambda Y^{\prime}+\frac{\lambda}{D} Y^{\prime \prime} \sum \frac{\partial X}{\partial \sigma} \frac{\partial X}{\partial z}\right] d z+\frac{D}{\lambda} Y^{\prime \prime} d \sigma\right\} \\
& +\left[\left(a Z+b Z^{\prime}\right) d z+\left(\alpha Z+\beta Z^{\prime}\right) d \sigma\right]\left\{\left[-\lambda Z^{\prime}+\frac{\lambda}{D} Z^{\prime \prime} \sum \frac{\partial X}{\partial \sigma} \frac{\partial X}{\partial z}\right] d z+\frac{D}{\lambda} Z^{\prime \prime} d \sigma\right\} .
\end{aligned}
$$

Ovvero, svolgendo i calcoli e semplificando:

$$
\sum d \xi d X=e d z^{2}+\left(f+f^{\prime}\right) d z d \sigma+g d \sigma^{2}=-b \lambda d z^{2}-\lambda \beta d z d \sigma .
$$

Tale valore, perù, di $\sum d \xi d X$ è subordinato, evidentemente, alla ipotesi che noi abbiamo fatto di porre uguale a zero l'espressione:

$$
\alpha\left(\frac{\partial a}{\partial \sigma}-\frac{\partial \alpha}{\partial z}\right)+\beta\left(\frac{\partial b}{\partial \sigma}-\frac{\partial \beta}{\partial z}\right)
$$

giacche $\mathrm{i}$ valori che noi abbiamo adoperati per $d X, d Y, d Z$ nel calcolare $\sum d \xi d X$, sono $i$ valori ricavati subordinatamente all'ipotesi anzidetta.

Vediamo, invece, che espressione si sarebbe ottenuta prescindendo 
da essa; per questo adopereremo per $d \xi, d \eta, d \zeta$ i valori (4), e per le sommatorie $\sum X \frac{\partial X^{\prime}}{\partial z}$, etc. che vengono a figurare nello sviluppo di $\sum\left(\frac{\partial X}{\partial z} d z+\frac{\partial X}{\partial \sigma} d \sigma\right) d \xi$ le espressioni:

$$
\begin{aligned}
& \sum X \frac{\partial X^{\prime}}{\partial z}=\frac{1}{a \beta-\alpha b}\left[a\left(\frac{\partial a}{\partial \sigma}-\frac{\partial \alpha}{\partial z}\right)+b\left(\frac{\partial b}{\partial \sigma}-\frac{\partial \beta}{\partial z}\right)\right]=-\sum X^{\prime} \frac{\partial X}{\partial z}, \\
& \sum X \frac{\partial X^{\prime}}{\partial \sigma}=\frac{1}{a \beta-\alpha b}\left[\alpha\left(\frac{\partial a}{\partial \sigma}-\frac{\partial \alpha}{\partial z}\right)+\beta\left(\frac{\partial b}{\partial \sigma}-\frac{\partial \beta}{\partial z}\right)\right]=-\sum X^{\prime} \frac{\partial X}{\partial \sigma} .
\end{aligned}
$$

Avremo allora

$$
\begin{aligned}
\text { (k) } \sum d \xi d X= & -\frac{b}{a \beta-b \alpha}\left[a\left(\frac{\partial a}{\partial \sigma}-\frac{\partial \alpha}{\partial z}\right)+b\left(\frac{\partial b}{\partial \sigma}-\frac{\partial \beta}{\partial z}\right)\right] d z^{2}-\left(\frac{\partial a}{\partial \sigma}-\frac{\partial \alpha}{\partial z}\right) d z d \sigma \\
& +\frac{\beta}{a \beta-b \alpha}\left[\alpha\left(\frac{\partial a}{\partial \sigma}-\frac{\partial \alpha}{\partial z}\right)+\beta\left(\frac{\partial b}{\partial \sigma}-\frac{\partial \beta}{\partial z}\right)\right] d \sigma^{2}
\end{aligned}
$$

e questa seconda espressione di $\sum d \xi d X$ diventa, difatti, coincidente con l'altra, se si pone

$$
\alpha\left(\frac{\partial a}{\partial \sigma}-\frac{\partial \alpha}{\partial z}\right)+\beta\left(\frac{\partial b}{\partial \sigma}-\frac{\partial \beta}{\partial z}\right)=0 .
$$

Tale ipotesi assume, in conseguenza, un'importante interpretazione geometrica; e di fatti, consideriamo la linea di stringimento delle rigate della congruenza $X, Y, Z$; la distanza $r$ del piede della minima distanza dalla superficie iniziale della congruenza, sappiamo essere data dalla formola :

$$
r=-\frac{e d z^{2}+\left(f+f^{\prime}\right) d z d \sigma+g d \sigma^{2}}{E_{\mathrm{x}} d z^{2}+2 F_{\mathrm{x}} d z d \sigma+G_{\mathrm{z}} d \sigma^{2}}
$$

Se si vuole che la linea di stringimento cada sullia superficie iniziale, dovrà essere $r=0$, ossia zero la $2^{\mathrm{a}}$ forma fondamentale

ossia

$$
e d z^{2}+\left(f+f^{\prime}\right) d z d \sigma+g d \sigma^{2},
$$

$$
\begin{gathered}
-\frac{b}{a \beta-b \alpha}\left[a\left(\frac{\partial a}{\partial \sigma}-\frac{\partial \alpha}{\partial z}\right)+b\left(\frac{\partial b}{\partial \sigma}-\frac{\partial \beta}{\partial z}\right)\right]-\left(\frac{\partial a}{\partial \sigma}-\frac{\partial \alpha}{\partial z}\right) d z d \sigma \\
+\frac{\beta}{a \beta-\alpha b}\left[\alpha\left(\frac{\partial a}{\partial \sigma}-\frac{\partial \alpha}{\partial z}\right)+\beta\left(\frac{\partial b}{\partial \sigma}-\frac{\partial \beta}{\partial z}\right)\right] d \sigma^{2}=0 .
\end{gathered}
$$

Ora se facciamo

$$
\alpha\left(\frac{\partial a}{\partial \sigma}-\frac{\partial \alpha}{\partial z}\right)+\beta\left(\frac{\partial b}{\partial \sigma}-\frac{\partial \beta}{\partial z}\right)=0,
$$


la $2^{\mathbf{a}}$ forma fondamentale diviene, come abbiamo visto:

$$
\sum d \xi d X=-b \lambda d z^{2}-\beta \lambda d z d \sigma
$$

e ponendola uguale a zero si avri:

(m)

$$
b d z^{2}+\beta d z d \sigma=0
$$

che si scinde nelle due:

$$
z=\text { cost., } \quad b d z+\beta d \sigma=0 .
$$

L'equazione ( $m$ ) definisce le due famiglie di rigate the hanno le linee di stringimento sulla superficie iniziale della congruenza, ossia sulla superficie $S_{\mathrm{t}}$ applicabile sulla $S$ data.

Tali due famiglie di rigate saranno tangenti alla $S_{1}$ secondo le due famiglie di linee:

$$
\begin{gathered}
z=\text { cost. } \\
b d z+\beta d \sigma=0,
\end{gathered}
$$

che risulteranno loro linee di stringimento.

Dunque l'ipotesi

$$
\alpha\left(\frac{\partial a}{\partial \sigma}-\frac{\partial \alpha}{\partial z}\right)+\beta\left(\frac{\partial b}{\partial \sigma}-\frac{\partial \rho}{\partial z}\right)=0
$$

fa si che le linee di stringimento delle due famiglie di rigate della congruenza $X, Y, Z$ per cui tali linee cadono sulla superficie $S_{\mathrm{r}}$, siano per una famiglia il sistema $z=$ cost. e per l'altra le linee definite dall'equazione:

$$
b d z+\beta d \sigma=0 .
$$

Supponiamo ora che la superficie $S_{\mathrm{t}}$ si deformi in modo continuo, portandosi sulla superficie $S$ su cui la supponiamo applicabile, e trascinando seco nei suoi piani tangenti le rette della congruenza $X, Y, Z$ che abbiamo visto tangenti alla superficie $S_{\mathrm{s}}$ nei punti delle linee $z=$ cost.

Ricordando che l'angolo che tali rette fanno con dette linee è funzione unicamente dell'elemento lineare, facilmente si scorge come la deformazione della $S_{\mathrm{s}}$ porta, di conseguenza, una deformazione nelle rigate delia congruenza, che lascia inalterato il loro elemento lineare: in altri termini, a deformazione compiuta risulteranno tali rigate applicabili sulle loro deformate.

Per di più, dalla teoria delle superficie rigate è noto che se si deforma una di queste superficie in guisa che le generatrici rimangano 
rettilinee, la linea di stringimento si conserva ancora tale per la deformata.

Simile conclusione potremo, evidentemente, farla per le rigate della nostra congruenza $X, Y, Z$, allorchè questa si deforma trascinata dalla superficie $S_{\mathrm{x}}$ nel movimento di questa per portarsi sulla superficie $S$. Ma v'ha di più.

L'equazione che prima ci ha fornito le linee di stringimento per la congruenza $X, Y, Z$ era la

$$
b d z^{2}+\beta d z d \sigma=0,
$$

ma è ovvio riconoscere che dopo la deformazione essa è rimasta invariata, i coefficienti $b$ e $\beta$ dipendendo soltanto dall'elemento lineare, conservato nella deformazione.

Dunque, non solo le curve che sulle rigate erano linee di stringimento si conservano ancora tali dopo la deformazione, ma esse sono ancora sulla superficie $S$ quelle che erano sulla superficie $S_{1}$, ossia le curve $z=$ cost. e le curve definite dalla equazione $b d z+\beta d \sigma=0$.

Fermiamoci alle curve $z=$ cost.

Supponiamo che la forma dell'elemento lineare sia per la superficie $S$ una forma ridotta del Weingarten:

$$
d s^{2}=\left(a^{2}+b^{2}\right) d z^{2}+2(a \alpha+b \beta) d z d \sigma+\left(\alpha^{2}+\beta^{2}\right) d \sigma^{2},
$$

sarà : $\sigma=\Delta(z)$.

Consideriamo sulla superficie $S$ una congruenza formata da rette tangenti ad essa nei punti delle linee $z=$ cost. e facenti con queste linee un angolo funzione arbitraria dell'elemento lineare di $S$.

Sarà sempre possibile soddisfare alla condizione che le linee $z=$ cost. siano linee di stringimento per quelle rigate; in tal guisa sarà determinata la funzione anzidetta.

Deformandosi la superficie $S$ sino a portarsi su di una superficie $S_{\mathrm{r}}$ su cui sia applicabile, si deformerà la congruenza, ma, per quanto precede, essa congruenza sarà applicabile sulla deformata; le linee di stringimento si conserveranno quelle di prima, saranno del pari sulla $S_{1}$, e su questa saranno ancora le linee $z=$ cost.

E però, in luogo della superficie $S$ potremo considerare la congruenza delle rette $X, Y, Z$; ed in luogo della $S_{1}$ la congruenza deformata.

Resta a vedere come potremo costruire quest'ultima, data la prima. Rend. Circ. Matem. Palermo, t. XVI (1902). - Stampato il 9 ottobre Igor. 
Il mezzo di conseguir ciò ce lo di l'equazione fondamentale del WEINGARTEN.

Integrata tale equazione, avremo una famiglia di curve $z=$ cost. giacente su di una deformata della superficie $S$.

Per ogni punto di una di tali curve avremo anche determinata la curva $\sigma=\Delta(z)=$ cost.; ci sarì, quindi, possibile costrurre la retta della congruenza, sapendo che essa deve fare con la tangente alla $z=$ cost. un angolo funzione determinata dell'elemento lineare della superficie $S$.

Il Darboux, nel volume $2^{\circ}$ della sua a Théorie générale des surfaces", ha fatto vedere come lo studio di una superficie può farsi dipendere da quello del movimento su di essa di un triedro $(T)$ di cui il vertice posa sulla superficie, lo spigolo $z$ coincide con la normale ad essa, e $\mathrm{i}$ due spigoli $x$ ed $y$ giacciono sul piano tangente.

Siano $p, q, r$ le componenti della rotazione secondo i tre assi del triedro $(\mathrm{T})$ e $\xi, n$ le componenti della traslazione secondo gli assi $x$ ed $y$, allorchè varia soltanto la linea coordinata $u ; p_{1}, q_{1}, r_{1}$ le componenti della rotazione secondo $i$ tre assi, e $\xi_{1}, n_{1}$ quelle della traslazione secondo gli assi $x$ ed $y$, quando varia soltanto la linea coordinata $v$.

L'espressione del quadrato dell'elemento lineare risulta:

$$
d s^{2}=\left(\xi^{2}+n^{2}\right) d u^{2}+2\left(\xi \xi_{1}+n n_{1}\right) d u d v+\left(\xi_{1}^{2}+n_{1}^{2}\right) d v^{2} ;
$$

inoltre sussistono le relazioni:

dalle quali si ottiene:

$$
\begin{aligned}
& \frac{\partial \xi}{\partial \sigma}-\frac{\partial \xi}{\partial z}=n r_{1}-r n_{1}, \\
& \frac{\partial n}{\partial \sigma}-\frac{\partial n_{1}}{\partial z}=r \xi_{1}-\xi r_{1} ;
\end{aligned}
$$

(n) $\xi_{1}\left(\frac{\partial \xi}{\partial \sigma}-\frac{\partial \xi}{\partial z}\right)+n_{1}\left(\frac{\partial n}{\partial \sigma}-\frac{\partial n_{1}}{\partial z}\right)=r_{1}\left(\xi_{1} n-n_{1} \xi\right)$.

Se facciamo le posizioni:

la nostra ipotesi

$$
\begin{array}{ll}
a=\xi, & b=x_{1}, \\
\alpha=\xi_{1}, & \beta=r_{1},
\end{array}
$$

$$
\alpha\left(\frac{\partial a}{\partial \sigma}-\frac{\partial \alpha}{\partial z}\right)+\beta\left(\frac{\partial b}{\partial \sigma}-\frac{\partial \beta}{\partial z}\right)=0
$$

diviene identica alla espressione ( $\mathrm{n}$ ) quando in essa si ponga $r_{\mathrm{s}}=0$. 
(Si noti che $\zeta_{1} n-\zeta_{n}$ non può essere zero, altrimenti tale sarebbe $E G-F^{2}$ ).

Identificando, perciò, le nostre funzioni $a, b, \alpha, \beta$ rispettivamente con $\xi, n, \xi_{1}, n_{1}$ potremo dire che la nostra ipotesi

$$
x\left(\frac{\partial a}{\partial \sigma}-\frac{\partial x}{\partial z}\right)+\beta\left(\frac{\partial b}{\partial \sigma}-\frac{\partial \beta}{\partial z}\right)=0
$$

ha il significato cinematico di porre $r_{\mathrm{s}}=0$.

Ora la componente della rotazione del triedro $(T)$ intorno alla normale è, come si sa,

e, nel caso nostro,

$$
r d u+r_{1} d v
$$

$$
r d z+r_{1} d \sigma \text {. }
$$

L'equazione (vedi Darboux, volume IV, pag. 343):

$$
r d z+r_{1} d \sigma=0
$$

definisce una delle due famiglie di linee di stringimento delle superficie rigate generate dali'asse delle $x$ del triedro (T), ed è facile vedere come l'ipotesi $r_{\mathrm{I}}=0$ porta ad essere tali linee di stringimento le curve

$$
z=\text { cost. }
$$

Abbiamo, cosi, una conferma di quanto gia si disse sull'ipotesi

$$
x\left(\frac{\partial a}{\partial \sigma}-\frac{\partial \alpha}{\partial z}\right)+\beta\left(\frac{\partial b}{\partial \sigma}-\frac{\partial \beta}{\partial z}\right)=0 .
$$

Consideriamo ora finalmente l'equazione :

$$
b d z+\beta d \sigma=0,
$$

che, come si disse, definisce le curve della seconda famiglia di linee di stringimento.

Constatata con l'ipotesi $r_{1}=0$ l'esistenza della identita $b=n$, $\beta=n_{\mathbf{x}}$ potremo dire che tale altra famiglia di curve $\dot{e}$ formata da quelle linee spostandosi lungo le quali la componente della traslazione del triedro (T) lungo l'asse delle $y$ è nulla, mentre il Darboux aveva già trovato che per la famiglia $z=$ cost. era nulla la componente della rotazione del triedro intorno alla normale.

Roma, giugno rgor.

UBALDO BARBIERI. 